\title{
BOUNDS ON THE RATE OF CONVERGENCE FOR ONE CLASS OF INHOMOGENEOUS MARKOVIAN QUEUEING MODELS WITH POSSIBLE BATCH ARRIVALS AND SERVICES
}

\author{
AlEXANDER ZEIFMAN ${ }^{a, b, *}$, ROSTISLAV RAZUMCHIK ${ }^{d, e}$, YACOV SATIN $^{a}$, \\ KSENIA KISELEVA ${ }^{e, a}$, ANNA KOROTYSHEVA ${ }^{a}$, Victor KOROLEV ${ }^{c, d}$ \\ ${ }^{a}$ Department of Applied Mathematics \\ Vologda State University, S. Orlova 6, Vologda, Russia \\ e-mail: \{a_zeifman, yacovi, a_korotysheva, ksushakiseleva\}@mail.ru \\ ${ }^{b}$ Institute of Socio-Economic Development of Territories \\ Russian Academy of Sciences, 56A Gorky Street, Vologda, Russia \\ ${ }^{c}$ Faculty of Computational Mathematics and Cybernetics \\ Lomonosov Moscow State University, Leninskie Gory, Moscow, Russia \\ e-mail: victoryukorolev@yandex.ru \\ ${ }^{d}$ Institute of Informatics Problems \\ Russian Academy of Sciences, Vavilova 44-2, Moscow, 119333, Russia \\ e-mail: rrazumchik@ipiran.ru \\ ${ }^{e}$ Applied Probability and Informatics Department \\ Peoples' Friendship University of Russia (RUDN University), 6 Miklukho-Maklaya, Moscow, 117198, Russia \\ e-mail: razumchik_rv@rudn.university
}

\begin{abstract}
In this paper we present a method for the computation of convergence bounds for four classes of multiserver queueing systems, described by inhomogeneous Markov chains. Specifically, we consider an inhomogeneous $M / M / S$ queueing system with possible state-dependent arrival and service intensities, and additionally possible batch arrivals and batch service. A unified approach based on a logarithmic norm of linear operators for obtaining sharp upper and lower bounds on the rate of convergence and corresponding sharp perturbation bounds is described. As a side effect, we show, by virtue of numerical examples, that the approach based on a logarithmic norm can also be used to approximate limiting characteristics (the idle probability and the mean number of customers in the system) of the systems considered with a given approximation error.
\end{abstract}

Keywords: inhomogeneous birth and death processes, weak ergodicity, rate of convergence, sharp bounds, logarithmic norm, forward Kolmogorov system.

\section{Introduction}

In this paper we consider a class of Markov processes, which may be used to describe the evolution of the total number of customers in inhomogeneous Markov queueing systems. Let the system's state space be $\mathcal{X}=$ $\{0,1,2 \ldots\}$, where the state $(i)$ means that there are a total of $i$ customers in the system. Throughout the paper it

${ }^{*}$ Corresponding author is assumed that all possible transition intensities between states are non-random functions of time and may depend on the system state.

There are two common problems related to such systems. The first one constitutes the computation of a time-dependent distribution of state probabilities and a limiting distribution (for example, in the case of periodic intensities). The second one is the computation of the rate of convergence and perturbation bounds. 
This paper deals with the second problem. Here we consider the following types of Markovian queueing systems:

I. possible state-dependent arrival and service intensities,

II. state-independent batch arrivals and a statedependent service intensity,

III. a possible state-dependent arrival intensity and a state-independent batch service,

IV. state-independent batch arrivals and a batch service.

For these four system types we describe a unified approach based on a logarithmic norm of linear operators, which allows us to obtain sharp upper and lower bounds on the rate of convergence and sharp perturbation bounds.

This unified approach has already been successfully applied to systems of classes I and IV. Specifically, for the inhomogeneous $M / M / 1$ system with state-dependent arrival and service intensities, as well as for the state-independent inhomogeneous $M / M / S$ system, the bounds were first obtained by Zeifman (1995b) and Doorn et al. (2010). Systems belonging to class IV have been studied in a number of papers (see, for example, Nelson et al., 1987; Li and Zhang, 2016; Chen et al., 2010) and the results related to convergence have been also obtained by Satin et al. (2013) and Zeifman et al. (2014a). Here we demonstrate that the approach is also suitable for systems from classes II and III, and thus offers a unified way toward an analysis of ergodicity properties of such Markov chains.

The approach is based on the special properties of linear systems of differential equations with non-diagonally non-negative matrices. Specifically, if the column-wise sums of the elements of this matrix are identical and equal to, say, $-\alpha^{*}(t)$, then the exact upper bound of order $\exp \left(-\int_{0}^{t} \alpha^{*}(u) \mathrm{d} u\right)$ can be obtained for the rate of convergence of the solutions of the system in the corresponding metric. Moreover, if the column-wise sums of the absolute values of the elements of this matrix are identical and equal to, say, $\chi^{*}(t)$, then the exact lower bound of order $\exp \left(-\int_{0}^{t} \chi^{*}(u) \mathrm{d} u\right)$ can be obtained for the convergence rate as well. The bounds are obtained in three steps. In the first step we exclude the (0) state from the forward Kolmogorov system of differential equations and thus obtain a new system with a new intensity matrix which is, in general, not non-diagonally non-negative. The second step is to transform the new intensity matrix in such a way that non-diagonally elements are non-negative and which leads to (loosely speaking) the least distance between specifically defined upper and lower bounds. The third step uses the logarithmic norm for the estimation of the convergence rate.
Here the key step is the second one. The transformation is made using a sequence of positive numbers $\left\{d_{i}, i \geq 1\right\}$, which does not have any probabilistic sense and can be considered an analogue of Lyapunov functions. For a detailed discussion on application of the logarithmic norm and related techniques we can refer the reader to the series of papers by Doorn et al. (2010), Granovsky and Zeifman (2004), Zeifman (1995b), Zeifman et al. (2006; 2014a), Zeifman and Korolev (2015), Mitrophanov (2005b; 2004; 2005a), Kim et al. (2014), Kamiński (2015), Moiseev and Nazarov (2016a; 2016b) or Whitt $(1991 ; 2015)$. The advantage of this three-step approach is that it allows us to deal with time-homogeneous and time-inhomogeneous processes and leads to both upper and lower exact bounds to the convergence rate. In the time-homogeneous case (of the four classes of systems introduced above), the approach allows us to obtain the corresponding bounds to the decay parameter and gives an explicit bounds in the total variation norm (see Theorem 2).

The proposed approach also allows us to address the problem of computation of the limiting distribution of inhomogeneous Markov chains from a different perspective. In general, there are several approaches, which allow us to obtain more or less accurate solutions. These are the exact and approximate numerical solutions of the system of differential equations, approaches assuming piecewise constant parameters, and those based on modified system characteristics. For a review of many results, we can refer the reader to Schwarz et al. (2016). Although using the proposed approach we cannot determine the state probabilities as functions of time $t$, it is possible to compute approximately the limiting distribution while having analytically computable expressions for the approximation errors. Using truncation techniques, which were developed by Zeifman et al. (2006; 2014b), we present the results of the computation of the limiting characteristics in inhomogeneous $M / M / S$ systems of each of the four classes described above. The most interesting insight from the experiments is the following: Choose the arrival and service intensities in inhomogeneous $M / M / S$ systems (class I). Then, if we use these intensities (after a certain modification allowing bulk arrivals and group services) in inhomogeneous $M / M / S$ systems from class II, III or IV, the limiting mean numbers of customers for both the systems coincide, while idle probabilities do not.

The paper is structured as follows. In the next section a general description of the system under consideration is given and the necessary notation is introduced. Section 3 contains the main result of the paper, i.e., the theorem which specifies the convergence bounds. Section 4 provides explicit expressions for functions needed to compute convergence bounds. In the last two sections we provide extensive numerical examples and give some 
directions of further research.

\section{System description and definitions}

Consider an integer-valued time-dependent random variable $X(t)$ which signifies the total number of customers at time $t$ in a Markovian queueing system. Then the process $\{X(t), t \geq 0\}$ is a (possibly inhomogeneous) continuous-time Markov chain with state space $\mathcal{X}=\{0,1,2 \ldots\}$. Denote by $p_{i j}(s, t)=P\{X(t)=j \mid X(s)=i\}$, $i, j \geq 0,0 \leq s \leq t$, the transition probabilities of $X(t)$ and by $p_{i}(t)=P\{X(t)=i\}$ the probability that the Markov chain $\{X(t), t \geq 0\}$ is in state $i$ at time $t$. Let $\mathbf{p}(t)=\left(p_{0}(t), p_{1}(t), \ldots\right)^{T}$ be a probability distribution vector at instant $t$. Throughout the paper we assume that in each time interval of length $h$ the possible transitions and their associated probabilities are

$$
p_{i j}(t, t+h)= \begin{cases}q_{i j}(t) h+\alpha_{i j}(t, h) & \text { if } j \neq i, \\ 1+q_{i i}(t) h+\alpha_{i}(t, h) & \text { if } j=i,\end{cases}
$$

where all $\alpha_{i}(t, h)$ are $o(h)$ uniformly in $i$, that is to say, $\sup _{i}\left|\alpha_{i}(t, h)\right|=o(h)$ and

$$
q_{i i}(t)=-\sum_{k \in \mathcal{X}, k \neq i} q_{i k}(t) .
$$

Applying the standard approach developed by Granovsky and Zeifman (2004), Zeifman (1995b) and Zeifman et al. (2006), it is assumed that all the intensity functions $q_{i j}(t)$ are locally integrable on $[0, \infty)$.

The matrix $Q(t)=\left(q_{i j}(t)\right)_{i, j=0}^{\infty}$ is the intensity matrix of the Markov chain $\{X(t), t \geq 0\}$. Henceforth it is assumed that $Q(t)$ is essentially bounded, i.e.,

$$
\sup _{i}\left|q_{i i}(t)\right|=L(t) \leq L<\infty
$$

for almost all $t \geq 0$.

Probabilistic dynamics of the process $\{X(t), t \geq 0\}$ are given by the forward Kolmogorov system

$$
\frac{\mathrm{d}}{\mathrm{d} t} \mathbf{p}(t)=A(t) \mathbf{p}(t)
$$

where $A(t)=Q^{T}(t)$ is the transposed intensity matrix.

Throughout the paper by $\|$. $\|$ we denote the $l_{1}$-norm, i.e., $\|\mathbf{p}(t)\|=\sum_{k \in \mathcal{X}}\left|p_{k}(t)\right|$, and $\|Q(t)\|=$ $\sup _{j \in \mathcal{X}} \sum_{i \in \mathcal{X}}\left|q_{i j}\right|$. Let $\Omega$ be a set all stochastic vectors, i.e., $l_{1}$ vectors with non-negative coordinates and a unit norm. Hence we have $\|A(t)\|=2 \sup _{k \in \mathcal{X}}\left|q_{k k}(t)\right| \leq 2 L$ for almost all $t \geq 0$. Thus the operator function $A(t)$ from $l_{1}$ into itself is bounded for almost all $t \geq 0$ and locally integrable on $[0 ; \infty)$. Therefore we can consider (3) a differential equation in the space $l_{1}$ with a bounded operator.
It is well known (see Daleckij and Krein, 1974) that the Cauchy problem for the differential equation (3) has a unique solution for an arbitrary initial condition, and $\mathbf{p}(s) \in \Omega$ implies $\mathbf{p}(t) \in \Omega$ for $t \geq s \geq 0$.

Denote by $E(t, k)=E(X(t) \mid X(0)=k)$ the conditional expected number of customers in the system at instant $t$, provided that initially (at instant $t=$ $0) k$ customers were present in the system. Then $\sum_{k \geq 0} E(t, k) p_{k}(0)$ is the unconditional expected number of customers in the system at instant $t$, given that the initial distribution of the total number of customers was $\mathbf{p}(0)$.

In order to obtain perturbation bounds, we consider a class of perturbed Markov chains $\{\bar{X}(t), t \geq 0\}$ defined on the same state space $\mathcal{X}$ as the original Markov chain $\{X(t), t \geq 0\}$, with the intensity matrix $\bar{A}(t)$ and the same restrictions as those imposed on $A(t)$. It is assumed that $\|\hat{A}(t)\|=\|A(t)-\bar{A}(t)\| \leq \varepsilon$, for almost all $t \geq 0$, which means the perturbations are assumed to be small.

Before proceeding to the derivation of the main results of the paper, we recall two definitions. Note that a Markov chain $\{X(t), t \geq 0\}$ is called weakly ergodic if $\left\|\mathbf{p}^{*}(t)-\mathbf{p}^{* *}(t)\right\| \rightarrow 0$ as $t \rightarrow \infty$ for any initial conditions $\mathbf{p}^{*}(0)$ and $\mathbf{p}^{* *}(0)$, where $\mathbf{p}^{*}(t)$ and $\mathbf{p}^{* *}(t)$ are the corresponding solutions of (3). A Markov chain $\{X(t), t \geq 0\}$ has the limiting mean $\varphi(t)$ if $\lim _{t \rightarrow \infty}(\varphi(t)-E(t, k))=0$ for any $k$.

\section{Main results}

Recall that we have introduced $A(t)$ as the transposed intensity matrix $Q(t)$. Thus it has the form

$$
A(t)=\left(\begin{array}{ccccc}
a_{00}(t) & a_{01}(t) & \cdots & a_{0 r}(t) & \ldots \\
a_{10}(t) & a_{11}(t) & \cdots & a_{1 r}(t) & \ldots \\
a_{20}(t) & a_{21}(t) & \cdots & a_{2 r}(t) & \ldots \\
\ldots \ldots \ldots \ldots \ldots \ldots & \ldots & \ldots \ldots & \ldots \\
a_{r 0}(t) & a_{r 1}(t) & \cdots & a_{r r}(t) & \ldots \\
\ldots \ldots \ldots \ldots \ldots & \ldots \ldots \ldots \ldots \ldots
\end{array}\right)
$$

where $a_{i i}(t)=-\sum_{k \in \mathcal{X}, k \neq i} a_{k i}(t)$. Since $p_{0}(t)=$ $1-\sum_{i=1}^{\infty} p_{i}(t)$ due to the normalization condition, we can rewrite the system (3) as follows:

$$
\frac{\mathrm{d}}{\mathrm{d} t} \mathbf{z}(t)=B(t) \mathbf{z}(t)+\mathbf{f}(t)
$$

where

$$
\begin{gathered}
\mathbf{f}(t)=\left(a_{10}(t), a_{20}(t), \ldots\right)^{T} \\
\mathbf{z}(t)=\left(p_{1}(t), p_{2}(t), \ldots\right)^{T}
\end{gathered}
$$




$$
\begin{gathered}
B(t) \\
=\left(\begin{array}{ccccc}
a_{11}-a_{10} & a_{12}-a_{10} & \cdots & a_{1 r}-a_{10} & \cdots \\
a_{21}-a_{20} & a_{22}-a_{20} & \cdots & a_{2 r}-a_{20} & \cdots \\
\ldots \ldots \ldots \ldots \ldots \ldots \ldots \ldots \ldots \ldots \ldots \ldots & \ldots \ldots \ldots \\
a_{r 1}-a_{r 0} & a_{r 2}-a_{r 0} & \cdots & a_{r r}-a_{r 0} & \cdots \\
\vdots & \vdots & \vdots & \vdots & \ddots
\end{array}\right) .
\end{gathered}
$$

Each entry of $B(t)$ depends on $t$; see a detailed discussion of this transformation by Granovsky and Zeifman (2004), Zeifman (1995b) and Zeifman et al. (2006). Let $\left\{d_{i}, i \geq\right.$ $1\}$ with $d_{1}=1$ be an increasing sequence of positive numbers. Set

$$
W=\inf _{i \geq 1} \frac{d_{i}}{i}
$$

and denote by $D$ the upper triangular matrix of the following form:

$$
D=\left(\begin{array}{cccc}
d_{1} & d_{1} & d_{1} & \cdots \\
0 & d_{2} & d_{2} & \cdots \\
0 & 0 & d_{3} & \cdots \\
\vdots & \vdots & \vdots & \ddots
\end{array}\right)
$$

Let $l_{1 D}$ be the corresponding space of sequences,

$$
\begin{aligned}
& l_{1 D}=\left\{\mathbf{z}(t)=\left(p_{1}(t), p_{2}(t), \cdots\right)^{T} \mid\right. \\
& \left.\|\mathbf{z}(t)\|_{1 D} \equiv\|D \mathbf{z}(t)\|_{1}<\infty\right\},
\end{aligned}
$$

and introduce also the auxiliary norm $\|\cdot\|_{1 E}$ defined as $\|\mathbf{z}(t)\|_{1 E}=\sum_{k=1}^{\infty} k\left|p_{k}(t)\right|$. Then in the $\|\cdot\|_{1 D}$ norm the following two inequalities hold:

$$
\begin{aligned}
& \|\mathbf{z}(t)\|_{1 D} \\
& =d_{1}\left|\sum_{i=1}^{\infty} p_{i}(t)\right|+d_{2}\left|\sum_{i=2}^{\infty} p_{i}(t)\right| \\
& \quad+d_{3}\left|\sum_{i=3}^{\infty} p_{i}(t)\right|+\ldots \\
& \geq\left(\left|\sum_{i=1}^{\infty} p_{i}(t)\right|+\left|\sum_{i=2}^{\infty} p_{i}(t)\right|+\left|\sum_{i=3}^{\infty} p_{i}(t)\right|+\ldots\right) \\
& \geq \frac{1}{2}\left(|| \sum_{i=1}^{\infty} p_{i}(t)|+| \sum_{i=2}^{\infty} p_{i}(t) \mid\right) \\
& \quad+\left(\left|\sum_{i=2}^{\infty} p_{i}(t)\right|+\left|\sum_{i=3}^{\infty} p_{i}(t)\right|\right)+\ldots \\
& \quad \geq \frac{1}{2} \sum_{i=1}^{\infty}\left|p_{i}(t)\right|=\frac{1}{2}\|\mathbf{z}(t)\|_{1},
\end{aligned}
$$

$$
\begin{aligned}
& \|\mathbf{z}(t)\|_{1 E}=\sum_{k=1}^{\infty} k\left|p_{k}(t)\right| \\
& =\sum_{k=1}^{\infty} \frac{k}{d_{k}} d_{k}\left|p_{k}(t)\right| \leq W^{-1} \sum_{k=1}^{\infty} d_{k}\left|p_{k}(t)\right| \\
& =W^{-1} \sum_{k=1}^{\infty} d_{k}\left|\sum_{i=k}^{\infty} p_{i}(t)-\sum_{i=k-1}^{\infty} p_{i}(t)\right| \\
& \leq W^{-1} \sum_{k=1}^{\infty} d_{k}\left(\left|\sum_{i=k}^{\infty} p_{i}(t)\right|+\left|\sum_{i=k-1}^{\infty} p_{i}(t)\right|\right) \\
& \leq \frac{2}{W} \sum_{k=1}^{\infty} d_{k}\left|\sum_{i=k}^{\infty} p_{i}(t)\right| \leq \frac{2}{W}\|\mathbf{z}(t)\|_{1 D} .
\end{aligned}
$$

Consider Eqn. (5) in the space $l_{1 D}$, where $B(t)$ and $\mathbf{f}(t)$ are locally integrable on $[0,+\infty)$. Let us compute the logarithmic norm of the operator function $B(t)$. The motivation behind this can be found in the work of Doorn et al. (2010), and detailed proofs are provided by Zeifman (1995a). Recall that the logarithmic norm of the operator function $B(t)$ is defined as

$$
\gamma(B(t))=\lim _{h \rightarrow+0} h^{-1}(\|I+h B(t)\|-1) .
$$

Denote by $V(t, s)=V(t) V^{-1}(s)$ the Cauchy operator of Eqn. (5). Then the following important inequality holds:

$$
e^{-\int_{s}^{t} \gamma(-B(u)) \mathrm{d} u} \leq\|V(t, s)\| \leq e^{\int_{s}^{t} \gamma(B(u)) \mathrm{d} u} .
$$

Further, for an operator function from $l_{1}$ to itself, we have the simple formula

$$
\gamma(B(t))=\sup _{j}\left(b_{j j}(t)+\sum_{i \neq j}\left|b_{i j}(t)\right|\right) .
$$

Moreover, for the logarithmic norm of the operator function $B(t)$ in the $\|\cdot\|_{1 D}$ norm, we have

$$
\gamma(B(t))_{1 D}=\gamma\left(D B(t) D^{-1}\right)_{1} .
$$

Denote by $b_{i j}^{*}(t)$ the elements of the matrix $D B(t) D^{-1}$, i.e., $D B(t) D^{-1}=\left(b_{i j}^{*}(t)\right)_{i, j=1}^{\infty}$. Assume that

$$
b_{i j}^{*}(t) \geq 0, \quad i \neq j, \quad t \geq 0 .
$$

Set

$$
\alpha_{i}(t)=\sum_{j=0}^{\infty} b_{j i}^{*}(t), \quad \chi_{i}(t)=-\sum_{j=0}^{\infty}\left|b_{j i}^{*}(t)\right|, i \geq 1
$$

and let $\alpha(t)$ and $\beta(t)$ denote respectively the least lower and the least upper bound of the sequence of functions $\left\{\alpha_{i}(t), i \geq 1\right\}$, and let $\chi$ denote the least upper bound of $\left\{\chi_{i}(t), i \geq 1\right\}$, i.e.,

$$
\alpha(t)=\inf _{i \geq 1} \alpha_{i}(t), \quad \beta(t)=\sup _{i \geq 1} \alpha_{i}(t),
$$




$$
\chi(t)=\sup _{i \geq 1} \chi_{i}(t) .
$$

Then the logarithmic norms of $B(t)$ and $(-B(t))$ are

$$
\begin{aligned}
& \gamma(B(t))_{1 D}=\sup _{i} \alpha_{i}(t)=-\alpha(t), \\
& \gamma(-B(t))_{1 D}=\sup \chi_{i}(t)=\chi(t) .
\end{aligned}
$$

If now we define $\mathbf{v}(t)=D\left(\mathbf{p}^{*}(t)-\mathbf{p}^{* *}(t)\right)$, then

$$
\frac{\mathrm{d}}{\mathrm{d} t} \mathbf{v}(t)=D B(t) D^{-1} \mathbf{v}(t) .
$$

Notice that, due to 11 , the inequality $\mathbf{v}(s) \geq 0$ implies that $\mathbf{v}(t) \geq \mathbf{0}$ for any $t \geq s$. Hence

$$
\frac{\mathrm{d}}{\mathrm{d} t} \sum_{i=1}^{\infty} v_{i}(t) \geq-\beta(t) \sum_{i=1}^{\infty} v_{i}(t),
$$

and we can establish the following theorem.

Theorem 1. Let there exist an increasing sequence $\left\{d_{j}, j \geq 1\right\}$ of positive numbers with $d_{1}=1$, such that (11) holds, and $\alpha(t)$ defined by (13) satisfies

$$
\int_{0}^{\infty} \alpha(t) \mathrm{d} t=+\infty
$$

Then the Markov chain $\{X(t), t \geq 0\}$ is weakly ergodic and the following bounds hold:

$$
\begin{aligned}
& e^{-\int_{s}^{t} \chi(u) \mathrm{d} u}\left\|\mathbf{p}^{*}(s)-\mathbf{p}^{* *}(s)\right\|_{1 D} \\
& \quad \leq\left\|\mathbf{p}^{*}(t)-\mathbf{p}^{* *}(t)\right\|_{1 D} \\
& \quad \leq e^{-\int_{s}^{t} \alpha(u) \mathrm{d} u}\left\|\mathbf{p}^{*}(s)-\mathbf{p}^{* *}(s)\right\|_{1 D},
\end{aligned}
$$

$$
\begin{aligned}
\| \mathbf{p}^{*}(t)- & \mathbf{p}^{* *}(t) \| \\
& \leq 4 e^{-\int_{s}^{t} \alpha(u) \mathrm{d} u}\left\|\mathbf{z}^{*}(s)-\mathbf{z}^{* *}(s)\right\|_{1 D}, \\
\| \mathbf{p}^{*}(t)- & \mathbf{p}^{* *}(t) \|_{1 E} \\
& \leq \frac{2}{W} e^{-\int_{s}^{t} \alpha(u) \mathrm{d} u}\left\|\mathbf{z}^{*}(s)-\mathbf{z}^{* *}(s)\right\|_{1 D},
\end{aligned}
$$

for any initial conditions $s \geq 0, \mathbf{p}^{*}(s), \mathbf{p}^{* *}(s)$ and any $t \geq s$.

$$
\begin{aligned}
& \text { If in addition } D\left(\mathbf{p}^{*}(s)-\mathbf{p}^{* *}(s)\right) \geq \mathbf{0} \text {, then } \\
& \begin{aligned}
\left\|\mathbf{p}^{*}(t)-\mathbf{p}^{* *}(t)\right\|_{1 D} \\
\quad \geq e^{-\int_{s}^{t} \beta(u) \mathrm{d} u}\left\|\mathbf{p}^{*}(s)-\mathbf{p}^{* *}(s)\right\|_{1 D},
\end{aligned}
\end{aligned}
$$

for any $0 \leq s \leq t$.

We can also obtain the corresponding perturbation bounds. The first results in this direction are given by Kartashov (1985; 1996), stronger results are stated by Mitrophanov (2003), and for the general approach one can refer to Zeifman and Korolev (2014). The respective uniform-in-time truncation bounds can be obtained via techniques proposed by Zeifman et al. (2014b; 2016a).

If the Markov chain is homogeneous, then no elements $b_{i j}^{*}(t)$ of the matrix $D B(t) D^{-1}$ depend on $t$, i.e., the quantities in (13) are constants. Thus, instead of the general bounds given by Theorem 1, we can specify and obtain the following theorem.

Theorem 2. Assume that there exists an increasing sequence $\left\{d_{j}, j \geq 1\right\}$ of positive numbers with $d_{1}=1$, such that (11) holds, and $\alpha(t)=\alpha$ defined by (13) is positive, i.e., $\alpha>0$. Then the Markov chain $\{X(t), t \geq 0\}$ is strongly ergodic and the following bounds hold:

$$
\begin{gathered}
e^{-\chi t}\left\|\mathbf{p}^{*}(0)-\mathbf{p}^{* *}(0)\right\|_{1 D} \\
\leq\left\|\mathbf{p}^{*}(t)-\mathbf{p}^{* *}(t)\right\|_{1 D} \\
\leq e^{-\alpha t}\left\|\mathbf{p}^{*}(0)-\mathbf{p}^{* *}(0)\right\|_{1 D} \\
\left\|\mathbf{p}^{*}(t)-\mathbf{p}^{* *}(t)\right\| \leq 4 e^{-\alpha t}\left\|\mathbf{z}^{*}(0)-\mathbf{z}^{* *}(0)\right\|_{1 D}, \\
\left\|\mathbf{p}^{*}(t)-\mathbf{p}^{* *}(t)\right\|_{1 E} \leq \frac{2}{W} e^{-\alpha t}\left\|\mathbf{z}^{*}(0)-\mathbf{z}^{* *}(0)\right\|_{1 D},
\end{gathered}
$$

for any initial conditions $s \geq 0, \mathbf{p}^{*}(0), \mathbf{p}^{* *}(0)$ and any $t \geq 0$. If, in addition, $D\left(\mathbf{p}^{*}(0)-\mathbf{p}^{* *}(0)\right) \geq \mathbf{0}$, then

$$
\left\|\mathbf{p}^{*}(t)-\mathbf{p}^{* *}(t)\right\|_{1 D} \geq e^{-\beta t}\left\|\mathbf{p}^{*}(0)-\mathbf{p}^{* *}(0)\right\|_{1 D},
$$

for any $t \geq 0$.

For the decay parameter $\alpha^{*}$ defined as

$$
\lim _{t \rightarrow \infty}\left(p_{i j}(t)-\pi_{j}\right)=O\left(e^{-\alpha^{*} t}\right),
$$

where $\left\{\pi_{j}, j \geq 0\right\}$ are the stationary probabilities of the chain, we have $\alpha^{*} \geq \alpha$.

Notice that some additional results related to Theorem 2 can also be found in the works of Doorn et al. (2010) or Granovsky and Zeifman (2000). If we assume that the intensities $q_{i j}(t)$ are 1-periodic in $t$, i.e., $q_{i j}(t)$ are periodic functions and the length of the period is equal to one, then the Markov chain $\{X(t), t \geq 0\}$ has the limiting 1-periodic limiting regime. Under the assumptions of Theorem 1, the Markov chain $\{X(t), t \geq 0\}$ is exponentially weakly ergodic. A detailed discussion of these results is provided by Zeifman et al. (2006).

In this section we present a slightly detailed analysis of two special cases: a homogeneous case and the case with periodic intensities. Firstly note that in both the cases there exist positive $R$ and $a$ such that

$$
e^{-\int_{s}^{t} \alpha(u) \mathrm{d} u} \leq R e^{-a(t-s)}
$$

for any $0 \leq s \leq t$. Hence the Markov chain $\{X(t), t \geq 0\}$ is exponentially weakly ergodic. Indeed, if $\{X(t), t \geq 0\}$ 
is a homogeneous Markov chain, then we may set $R=1$, $a=\alpha$ given by (13). If all the intensity functions $q_{i j}(t)$ are 1-periodic in $t$, then we may set

$$
\begin{aligned}
a & =\int_{0}^{1} \alpha(t) \mathrm{d} t, \\
R & =e^{K}, \\
K & =\sup _{|t-s| \leq 1} \int_{s}^{t} \alpha(u) \mathrm{d} u .
\end{aligned}
$$

By doing so, for any solution of (5), the following bound holds:

$$
\begin{aligned}
\|\mathbf{z}(t)\|_{1 D} \leq & \|V(t)\|_{1 D}\|\mathbf{z}(0)\|_{1 D} \\
& +\int_{0}^{t}\|V(t, \tau)\|_{1 D}\|\mathbf{f}(\tau)\|_{1 D} \mathrm{~d} \tau \\
\leq & R e^{-a t}\|\mathbf{z}(0)\|_{1 D}+\frac{F R}{a}
\end{aligned}
$$

where $F$ is such that $\|\mathbf{f}(t)\|_{1 D} \leq F$ for almost all $t \in$ $[0,1]$. Hence we have the upper bound for the limit

$$
\limsup _{t \rightarrow \infty}\|\mathbf{z}(t)\|_{1 D} \leq \frac{F R}{a}
$$

for any initial condition and

$$
\begin{aligned}
\left\|\mathbf{p}(0)-\mathbf{e}_{0}\right\|_{1 D} & =\|\mathbf{p}(0)\|_{1 D} \\
& =\|\mathbf{z}(0)\|_{1 D} \leq \limsup _{t \rightarrow \infty}\|\mathbf{z}(t)\|_{1 D},
\end{aligned}
$$

where $\mathbf{e}_{i}$ denotes the unit vector of zeros with one in the $i$-th place. If the initial distribution is $\mathbf{p}^{* *}(0)=\mathbf{e}_{0}$, then $\mathbf{z}^{* *}(0)=\mathbf{0}, \mathbf{z}(t) \geq 0$ for any $\mathbf{p}^{*}(0)$ and any $t \geq 0$. Therefore

$$
\begin{aligned}
\| \mathbf{z}(t) & \|_{1 D} \\
= & d_{1} p_{1}+\left(d_{1}+d_{2}\right) p_{2} \\
& +\left(d_{1}+d_{2}+d_{3}\right) p_{3}+\ldots \\
= & d_{1} p_{1}+\frac{d_{1}+d_{2}}{2} 2 p_{2}+\frac{d_{1}+d_{2}+d_{3}}{3} 3 p_{3}+\ldots \\
\geq & \inf _{k} \frac{d_{1}+\cdots+d_{k}}{k}\|\mathbf{z}(t)\|_{1 E},
\end{aligned}
$$

and we can use

$$
W^{*}=\inf _{k} \frac{d_{1}+\cdots+d_{k}}{k}
$$

instead of

$$
W=\inf _{k} \frac{d_{k}}{k}
$$

given by (7) in all the bounds on the rate of convergence. Finally, for the two special cases considered we have the following two corollaries.
Corollary 1. Let $\{X(t), t \geq 0\}$ be a homogeneous Markov chain and let there exist an increasing sequence $\left\{d_{j}, j \geq 1\right\}$ of positive numbers with $d_{1}=1$ such that (11) holds and, in addition, $\alpha>0$. Then $\{X(t), t \geq 0\}$ is exponentially ergodic and the following bounds hold:

$$
\begin{gathered}
\|\pi-\mathbf{p}(t, 0)\| \leq \frac{4 F}{\alpha} e^{-\alpha t}, \\
|\varphi-E(t, 0)| \leq \frac{F}{\alpha W^{*}} e^{-\alpha t},
\end{gathered}
$$

where $\pi=\left(\pi_{0}, \pi_{1}, \ldots\right)^{T}$ denotes the vector of stationary probabilities of $\{X(t), t \geq 0\}$ and $\varphi=\sum_{j=0}^{\infty} j \pi_{j}$ and $\mathbf{p}(0,0)=\mathbf{e}_{0}$.

Corollary 2. Assume that all the intensity functions of the Markov chain $\{X(t), t \geq 0\}$ are 1-periodic in $t$. Suppose that there exists an increasing sequence $\left\{d_{j}, j \geq 1\right\}$ of positive numbers with $d_{1}=1$ such that (11) holds and, in addition, $\int_{0}^{1} \alpha(t) \mathrm{d} t=a>0$. Then $\{X(t), t \geq 0\}$ is exponentially weakly ergodic and the following bounds hold:

$$
\begin{gathered}
\|\pi(t)-\mathbf{p}(t, 0)\| \leq \frac{4 F R}{a} e^{-a t}, \\
|\varphi(t)-E(t, 0)| \leq \frac{F R}{a W^{*}} e^{-a t},
\end{gathered}
$$

where $\pi(t)=\left(\pi_{0}(t), \pi_{1}(t), \ldots\right)^{T}$ denotes the vector of limiting probabilities of $\{X(t), t \geq 0\}$ and $\varphi(t)=$ $\sum_{j=0}^{\infty} j \pi_{j}(t)$ and $\mathbf{p}(0,0)=\mathbf{e}_{0}$.

If the state space of a Markov chain is finite, there are a number of special results (see Doorn et al., 2010; Granovsky and Zeifman, 2000; Zeifman et al., 2013).

Before moving to the next section, we briefly describe one possible procedure for finding $\pi(t)$ and $\varphi(t)$ in the case of 1-periodic in $t$ intensities. Firstly, we estimate the instant $t=t^{*}$ (using the ergodicity bounds), starting from which the solution of the forward Kolmogorov system (3) with the initial condition $X(0)$ is within a fixed $\epsilon>0$ from the limiting periodic probabilities. Then we estimate the size $n^{*}$ of the state space $\left\{0,1, \ldots, n^{*}\right\}$, which guarantees the desired approximation error on the interval $\left[0, t^{*}+1\right]$. Then we find the solution of the truncated system on the interval $\left[0, t^{*}+1\right]$, and eventually the values for $\pi(t)$ and $\varphi(t)$ on the interval $\left[t^{*}, t^{*}+1\right]$.

\section{Convergence bounds}

In order to apply the results of Theorems 1 and 2 and to obtain the convergence bounds for the system from classes I-IV, we have to know exact expressions for the functions $\alpha_{i}(t)$ and $\chi_{i}(t)$, given by (13) and (14). In this section we provide such expressions for $\alpha_{i}(t)$ and $\chi_{i}(t)$ in the case of a single server. Then we show how these expressions are changed when we switch to the multiple server case. 
4.1. Inhomogeneous $M / M / S$ queueing system with batch arrivals and state-dependent service intensities. Consider a queueing system $M / M / 1$ with time-dependent arrival and service intensities. The service discipline for this queue (and all other queues in the following subsections) can be FIFO, LIFO or random. Let $\lambda_{k}(t)$ be the arrival intensity of the batch, containing $k$ customers, at instant $t$, and $\mu_{k}(t)$ be the service intensity at instant $t$ if the total number of customers in the system is equal to $k$. Then the transposed intensity matrix has the form

$$
\begin{aligned}
& A(t)=\left(\begin{array}{cccc}
a_{00}(t) & \mu_{1}(t) & 0 & 0 \\
\lambda_{1}(t) & a_{11}(t) & \mu_{2}(t) & 0 \\
\lambda_{2}(t) & \lambda_{1}(t) & a_{22}(t) & \mu_{3}(t) \\
\lambda_{3}(t) & \lambda_{2}(t) & \lambda_{1}(t) & a_{33}(t) \\
\lambda_{4}(t) & \lambda_{3}(t) & \lambda_{2}(t) & \lambda_{1}(t) \\
\ldots & \vdots & \vdots & \vdots
\end{array}\right. \\
& \left.\begin{array}{cc}
0 & \cdots \\
0 & \cdots \\
0 & \cdots \\
\mu_{4}(t) & \cdots \\
a_{44}(t) & \cdots \\
\vdots & \ddots
\end{array}\right)
\end{aligned}
$$

where diagonal elements of $A(t)$ are such that all column sums are equal to zero for any $t \geq 0$. As the assumption (11) is fulfilled, we have

$$
\begin{aligned}
\alpha_{j}(t)= & \mu_{j}(t)-\frac{d_{j-1}}{d_{j}} \mu_{j-1}(t) \\
& +\sum_{i=1}^{\infty}\left(1-\frac{d_{i+j}}{d_{j}}\right) \lambda_{i}(t)
\end{aligned}
$$

and

$$
\begin{aligned}
\chi_{j}(t)= & \mu_{j}(t)+\frac{d_{j-1}}{d_{j}} \mu_{j-1}(t) \\
& +\sum_{i=1}^{\infty}\left(1+\frac{d_{i+j}}{d_{j}}\right) \lambda_{i}(t) .
\end{aligned}
$$

Therefore, Theorems 1 and 2 hold for the specified $\alpha_{i}(t)$ and $\chi_{i}(t)$.

Consider now a queueing system $M / M / S$ with $S>$ 1 servers, as well as time-dependent arrival and service intensities. Customers arrive at the system in batches of a size no greater than $S$. Assume that the arrival intensity of a batch, containing $k$ customers, at instant $t$ is equal to $\lambda_{k}(t)=\lambda(t) / S k$ if $1 \leq k \leq S$ and $\lambda_{k}(t)=0$ if $k>S$. Denote by $\mu_{k}(t)$ the service intensity at instant $t$ and assume that $\mu_{k}(t)=\min (k, S) \mu(t)$. For the assumed values of $\lambda_{k}(t)$ and $\mu_{k}(t)$, and the expressions for $\alpha_{i}(t)$ and $\chi_{i}(t)$ given above, Theorems 1 and 2 hold.
4.2. Inhomogeneous $M / M / S$ queueing system with batch service and state-dependent arrival intensities. Consider a queueing system $M / M / 1$ with time-dependent arrival and service intensities. But now let $\lambda_{k}(t)$ be the arrival intensity of $k$ customers at instant $t$ if the total number of customers in the system is equal to $k$, and $\mu_{k}(t)$ be the service intensity at instant $t$ of a group of $k$ customers. Then the transposed intensity matrix has the form

$$
\begin{aligned}
& A(t)=\left(\begin{array}{cccc}
a_{00}(t) & \mu_{1}(t) & \mu_{2}(t) & \mu_{3}(t) \\
\lambda_{0}(t) & a_{11}(t) & \mu_{1}(t) & \mu_{2}(t) \\
0 & \lambda_{1}(t) & a_{22}(t) & \mu_{1}(t) \\
0 & 0 & \lambda_{2}(t) & a_{33}(t) \\
\vdots & \vdots & \vdots & \vdots
\end{array}\right. \\
& \left.\begin{array}{cc}
\mu_{4}(t) & \cdots \\
\mu_{3}(t) & \cdots \\
\mu_{2}(t) & \cdots \\
\mu_{1}(t) & \cdots \\
\vdots & \ddots
\end{array}\right)
\end{aligned}
$$

where $a_{i i}(t)=-\sum_{k=1}^{i} \mu_{k}(t)-\lambda_{i}(t)$. Then Theorems 1 and 2 hold for

$$
\begin{aligned}
\alpha_{i}(t)= & \mu_{i}(t)-\sum_{k=1}^{i-1}\left(\mu_{i-k}(t)-\mu_{i}(t)\right) \frac{d_{k}}{d_{i}} \\
& +\lambda_{i-1}(t)-\frac{d_{i+1}}{d_{i}} \lambda_{i}(t), \\
\chi_{i}(t)= & \mu_{i}(t)+\sum_{k=1}^{i-1}\left(\mu_{i-k}(t)-\mu_{i}(t)\right) \frac{d_{k}}{d_{i}} \\
& +\lambda_{i-1}(t)+\frac{d_{i+1}}{d_{i}} \lambda_{i}(t) .
\end{aligned}
$$

Consider again a queueing system $M / M / S$ with $S>1$ servers and time-dependent arrival and service intensities. The customers are served in batches of a size no greater than $S$. Assume that the service intensity is $\mu_{k}(t)=\mu(t) / k$ if $1 \leq k \leq S$ and $\mu_{k}(t)=0$ if $k>S$. Denote by $\lambda(t)$ the arrival intensity. For the assumed values of $\lambda_{k}(t)$ and $\mu_{k}(t)$, and the expressions for $\alpha_{i}(t)$ and $\chi_{i}(t)$ given above, Theorems 1 and 2 remain valid.

4.3. Inhomogeneous $M / M / S$ queueing system with batch arrivals and service. Consider a queueing system $M / M / 1$ with time-dependent arrival and service intensities. Customers arrive in batches and are served in batches as well. Let $\lambda_{k}(t)$ and $\mu_{k}(t)$ be the arrival and service intensity of a group of $k$ customers. This queueing system has been extensively studied with respect to the rate of convergence, truncation and perturbation bounds by Satin et al. (2013) and Zeifman et al. (2014a). The 
transposed intensity matrix in this case has the following form:

$$
A(t)=\left(\begin{array}{ccccc}
a_{00}(t) & \mu_{1}(t) & \mu_{2}(t) & \mu_{3}(t) & \ldots \\
\lambda_{1}(t) & a_{11}(t) & \mu_{1}(t) & \mu_{2}(t) & \ldots \\
\lambda_{2}(t) & \lambda_{1}(t) & a_{22}(t) & \mu_{1}(t) & \ldots \\
\lambda_{3}(t) & \lambda_{2}(t) & \lambda_{1}(t) & a_{33}(t) & \ldots \\
& \vdots & \vdots & \vdots & \ddots
\end{array}\right),
$$

where

$$
a_{i i}(t)=-\sum_{k=1}^{i} \mu_{k}(t)-\sum_{k=1}^{\infty} \lambda_{k}(t) .
$$

Therefore, Theorems 1 and 2 hold for

$$
\begin{aligned}
\alpha_{i}(t)= & -a_{i i}(t)-\sum_{k=1}^{i-1}\left(\mu_{i-k}(t)-\mu_{i}(t)\right) \frac{d_{k}}{d_{i}} \\
& -\sum_{k \geq 1} \lambda_{k}(t) \frac{d_{k+i}}{d_{i}},
\end{aligned}
$$

and

$$
\begin{aligned}
\chi_{i}(t)= & -a_{i i}(t)+\sum_{k=1}^{i-1}\left(\mu_{i-k}(t)-\mu_{i}(t)\right) \frac{d_{k}}{d_{i}} \\
& +\sum_{k \geq 1} \lambda_{k}(t) \frac{d_{k+i}}{d_{i}} .
\end{aligned}
$$

Consider again a queueing system $M / M / S$ with $S>1$ servers, as well as time-dependent arrival and service intensities. Assume that the size of an arrival batch and a service batch does exceed $S$. Let the arrival intensity of $k$ customers at instant $t$ be equal to $\lambda_{k}(t)=\lambda(t) / S k$ if $1 \leq k \leq S$ and $\lambda_{k}(t)=0$ if $k>S$; let the service intensity be equal to $\mu_{k}(t)=\mu(t) / k$ if $1 \leq k \leq S$ and $\mu_{k}(t)=0$ if $k>S$. For the assumed values of $\lambda_{k}(t)$ and $\mu_{k}(t)$, and the above expressions for $\alpha_{i}(t)$ and $\chi_{i}(t)$, Theorems 1 and 2 remain valid.

4.4. Inhomogeneous $M / M / S$ queueing system with state-dependent arrival and service intensities. If in the queueing system $M / M / 1$ the arrival intensities $\lambda_{n}(t)$ and the service intensities $\mu_{n}(t)$ are time-dependent and also depend on the total number of customers $n$ in the system, then the queue-length process for a general Markovian queue is an inhomogeneous birth-death process with birth and death intensities equal to $\lambda_{n}(t)$ and $\mu_{n}(t)$, respectively. Thus Theorems 1 and 2 hold with

$$
\begin{aligned}
\alpha_{j}(t)= & \mu_{j}(t)-\frac{d_{j-1}}{d_{j}} \mu_{j-1}(t) \\
& +\lambda_{j-1}(t)-\frac{d_{j+1}}{d_{j}} \lambda_{j}(t)
\end{aligned}
$$

and

$$
\begin{aligned}
\chi_{j}(t)= & \mu_{j}(t)+\frac{d_{j-1}}{d_{j}} \mu_{j-1}(t) \\
& +\lambda_{j-1}(t)+\frac{d_{j+1}}{d_{j}} \lambda_{j}(t) .
\end{aligned}
$$

Consider again an ordinary queueing system $M / M / S$ with $S>1$ servers, and time-dependent arrival and service intensities $\lambda(t)$ and $\mu_{n}(t)=\min (n, S) \mu(t)$, respectively. For the assumed values of $\lambda_{k}(t), \mu_{k}(t)$, and the expressions for $\alpha_{i}(t)$ and $\chi_{i}(t)$ given above, Theorems 1 and 2 remain valid.

\section{Numerical examples}

The purposes of the numerical section are two-fold. Firstly, we demonstrate that the convergence bounds obtained in the previous section can indeed be computed. Having fixed the arrival and service intensities in the inhomogeneous $M / M / S$ queueing system with state-independent arrival and service intensities (class $I$ ), we specify the sequence $\left\{d_{i}, i \geq 1\right\}$ and provide the corresponding bounds using Corollary 2. Secondly, we show that the approach proposed in this paper can be used to compute approximations for the limiting characteristics of the systems with a given approximation error. The characteristics under consideration are the limiting idle probability and the limiting mean number of customers in the system.

The systems considered in this numerical section are as follows:

(i) inhomogeneous $M / M / 100$ queueing system with state-independent arrival and service intensities,

(ii) inhomogeneous $M / M / 100$ queueing system with state-independent batch arrivals,

(iii) inhomogeneous $M / M / 100$ queueing system with state-independent batch service,

(iv) inhomogeneous $M / M / 100$ queueing system with state-independent batch arrivals and batch service.

All transition intensities are assumed to be periodic functions of time. Customers in all four cases are served in FCFS order. The inhomogeneous $M / M / 100$ system consists of a single infinite capacity queue and 100 servers. We assume that the arrivals occur according to the inhomogeneous Poisson process with the intensity $\lambda^{*}(t ; i)$ equal to

$$
\lambda^{*}(t ; i)=i(1+\sin 2 \pi t), \quad i>0, t>0 .
$$

Whenever the server becomes free, the first customer from the queue (if there is any) enters the server. We will 
model the service times to be interarrival times of an inhomogeneous Poisson process with the intensity

$$
\mu^{*}(t)=3+\cos 2 \pi t
$$

This is a time-varying counterpart to the exponential distribution. The departure process will behave like this Poisson process when the queue length is non-zero.

In the inhomogeneous $M / M / 100$ queueing system with batch arrivals it is assumed that customers arrive in batches in accordance with an inhomogeneous Poisson process of intensity $\lambda^{*}(t ; i) \sum_{i=1}^{S}(S i)^{-1}$. The size of the arriving group is a random variable with discrete probability distribution $(S k)^{-1} / \sum_{i=1}^{S}(S i)^{-1}, 1 \leq k \leq$ $S$. The sizes and interarrival times of successive arriving groups are stochastically independent. Thus the total arrival intensity is $\lambda_{k}(t, i)=(S k)^{-1} \lambda^{*}(t, i)$ if $1 \leq k \leq S$ and $\lambda_{k}(t, i)=0$ if $k>S$. Whenever the server becomes free, a customer from the queue (if there is any) enters the server and gets served according to the exponential distribution with intensity $\mu_{k}(t)=\min (k, S) \mu^{*}(t)$.

In the inhomogeneous $M / M / 100$ queueing system with a batch service customers arrive in accordance with the inhomogeneous Poisson process of the same intensity $\lambda^{*}(t, i)=i(1+\sin 2 \pi t)$. But the service takes place in batches of a size no greater than $S$, and the service times are exponentially distributed with the service intensity equal to $\mu_{k}(t)=\mu^{*}(t) / k$ if $1 \leq k \leq S$ and $\mu_{k}(t)=0$ if $k>S$.

Finally, in the inhomogeneous $M / M / 100$ queueing system with batch arrivals and batch service, the total arrival intensity is $\lambda_{k}(t, i)=\lambda^{*}(t, i) / S k$ if $1 \leq k \leq S$ and $\lambda_{k}(t, i)=0$ if $k>S$, and the service intensity is $\mu_{k}(t)=\mu^{*}(t) / k$ if $1 \leq k \leq S$ and $\mu_{k}(t)=0$ if $k>S$.

Such a choice of the system parameters is made only for the purpose of illustration.

Let us find the convergence bounds in the case (i), i.e., for the inhomogeneous $M / M / 100$ queueing system with state-independent arrival and service intensities. Let $i=50$, i.e., let the arrival intensity be equal to

$$
\lambda^{*}(t ; 50)=50(1+\sin 2 \pi t), \quad t>0 .
$$

Specify the sequence $\left\{d_{i}, i \geq 1\right\}$ as follows:

- $d_{i}=1$, if $1 \leq i \leq 100$;

- $d_{101}=1.05 d_{100}, d_{102}=1.1 d_{101}, d_{103}=1.3 d_{102}$, $d_{104}=1.6 d_{103}, d_{105}=2 d_{104}$

- $d_{i}=2.3^{i-105} d_{105}$, if $i \geq 106$.

Such a sequence $\left\{d_{i}, i \geq 1\right\}$ guarantees that the assumptions of Corollary 2 are fulfilled and we have bounds on the rate of convergence to the limiting characteristics given by (32) and (33) with $a=1.7$, $R=2, F=100$. In order to approximate the

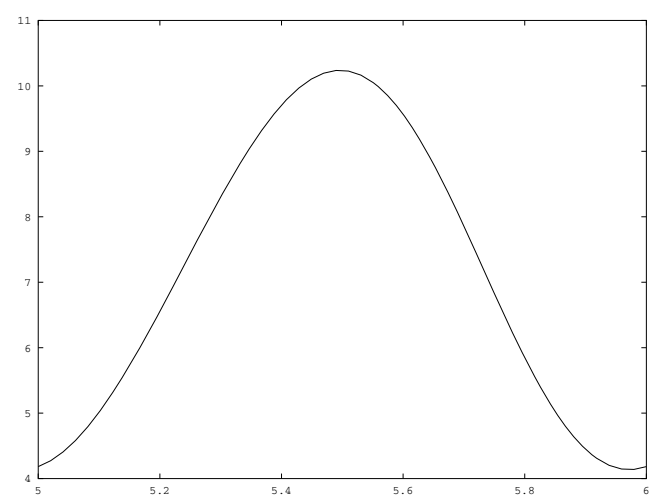

Fig. 1. Case (i): the arrival intensity is $\lambda^{*}(t ; 20)$; approximation of the limiting mean number $\varphi(t)$ of customers in the system for $t \in[5,6]$.

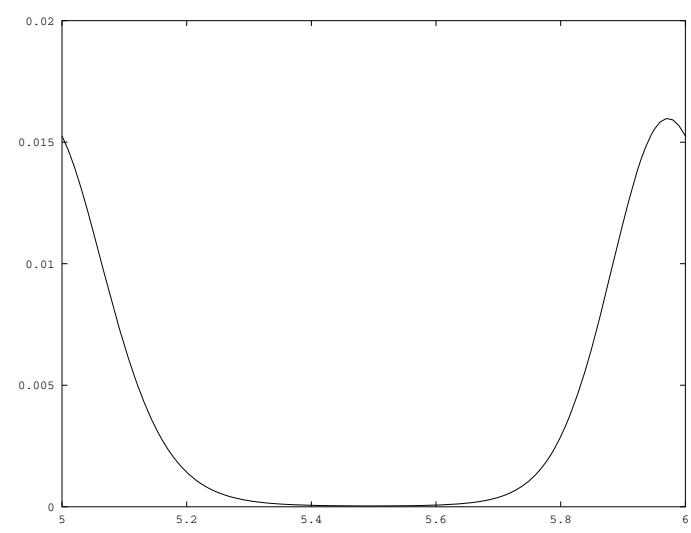

Fig. 2. Case (i): the arrival intensity is $\lambda^{*}(t ; 20)$; approximation of the limiting probability $p_{0}(t)$ of the empty queue for $t \in[5,6]$.

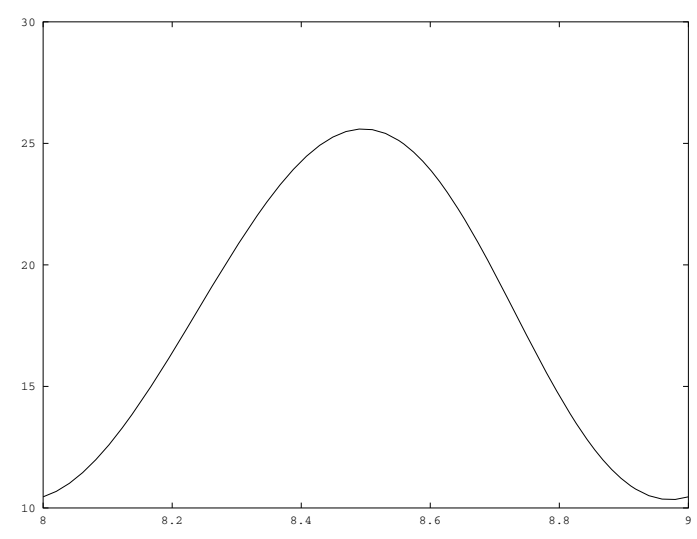

Fig. 3. Case (i): the arrival intensity is $\lambda^{*}(t ; 50)$; approximation of the limiting mean number $\varphi(t)$ of customers in the system for $t \in[5,6]$.

limiting characteristics for all four cases (i)-(iv), we can apply Theorems 5 and 8 from Zeifman et al. (2014b). 


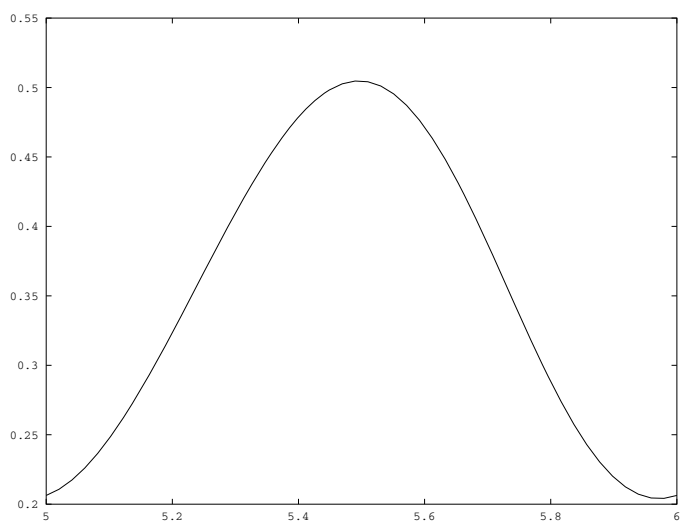

Fig. 4. Case (ii): the arrival intensity is $\lambda^{*}(t ; 10)$; approximation of the limiting mean number $\varphi(t)$ of customers in the system for $t \in[5,6]$.

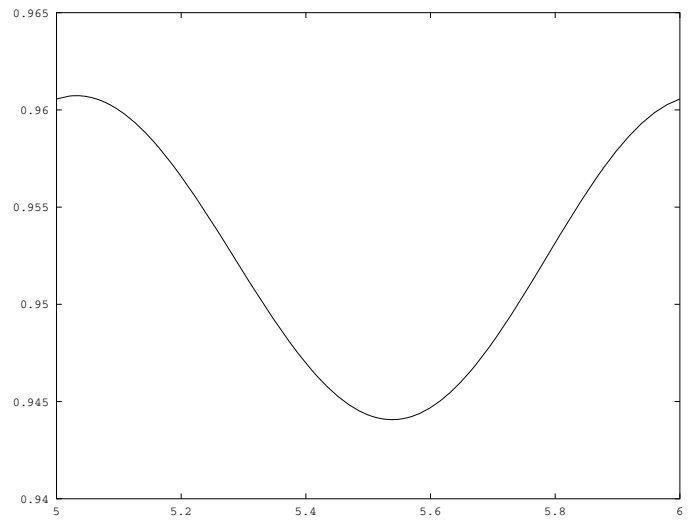

Fig. 5. Case (ii): the arrival intensity is $\lambda^{*}(t ; 10)$; approximation of the limiting probability $p_{0}(t)$ of the empty queue for $t \in[5,6]$.

But firstly we have to specify the value $i$ in the arrival intensity $\lambda^{*}(t ; i)$, because as the arrival intensity (and thus load) grows, a bigger state space is needed. Assume that $i \leq 50$, i.e., the maximum arrival intensity allowed is $\lambda^{*}(t ; 50)=50(1+\sin 2 \pi t)$. Then we can compute the solution of the forward Kolmogorov system (3) for the truncated process on the state space $\{0,1, \ldots, 155\}$ on the interval $\left[0, t^{*}+1\right]$ with the initial condition $X(0)=0$. Hence we find the limiting idle probability and limiting mean value on the interval $\left[t^{*}, t^{*}+1\right]$ with an error less than $10^{-4}$, where $t^{*}=5$ or $t^{*}=7$.

Figures 1-15 display the plots of the limiting probability of the empty queue $p_{0}(t)$ and the limiting mean number $\varphi(t)$ of customers in the system for each of the cases (i)-(iv).

Note from Figs. 1 and 2 that in the case of a high arrival intensity the limiting probability $p_{0}(t)$ of the empty queue here equals zero for most of the time.

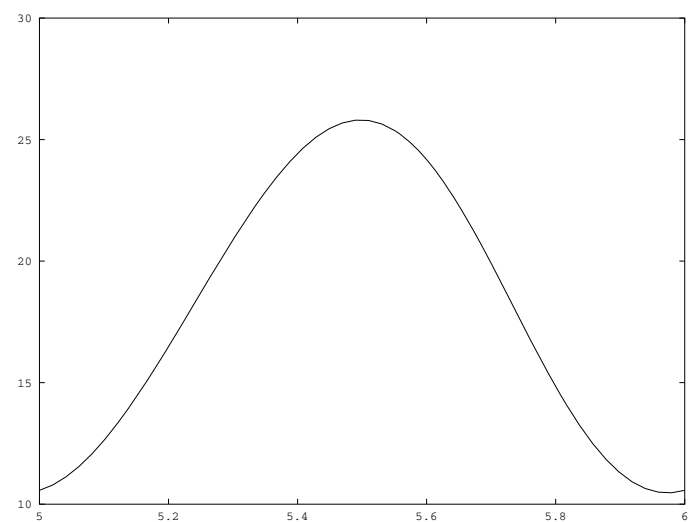

Fig. 6. Case (ii): the arrival intensity is $\lambda^{*}(t ; 50)$; approximation of the limiting mean number $\varphi(t)$ of customers in the system for $t \in[5,6]$.

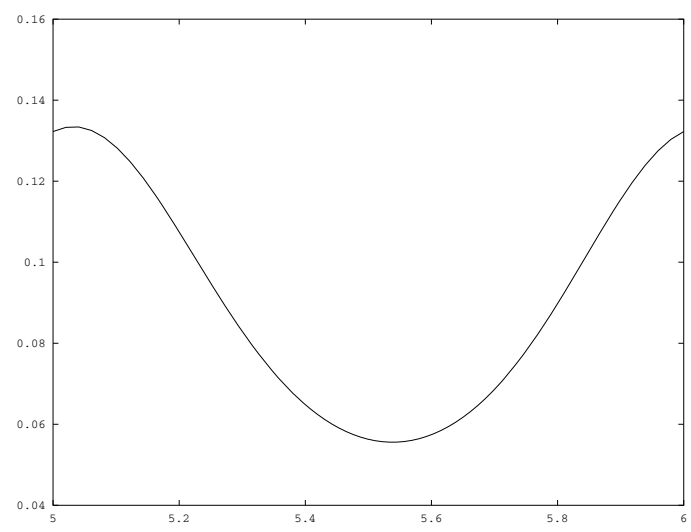

Fig. 7. Case (ii): the arrival intensity is $\lambda^{*}(t ; 50)$; approximation of the limiting probability $p_{0}(t)$ of the empty queue for $t \in[5,6]$.

\section{Conclusion}

From the presented figures we can see that the limiting mean number of customers in the system apparently does not depend on the system type, i.e., for all four different systems considered there is numerical evidence that the limiting means coincide. With respect to the probability of the empty queue, we observe clear dependence on the system type.

These numerical evidences show one of the directions of further research: explanation of these effects from an analytical point of view. Another direction is the generalization of the proposed method for non-Markovian systems and other types of inhomogeneous queueing systems (e.g., Almasi et al., 2005; Gudkova et al., 2016). One of the appealing candidates are queueing systems with balking, in which arrival intensities decrease with the growth of the total number of customers in the system. Yet another direction of research follows from the work 


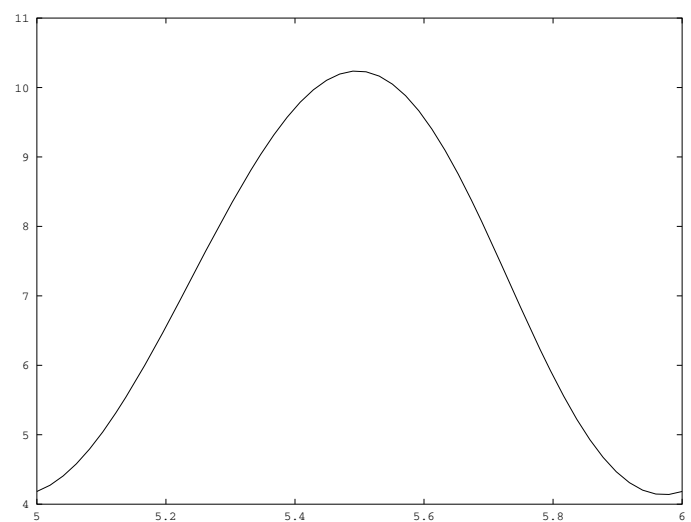

Fig. 8. Case (iii): the arrival intensity is $\lambda^{*}(t ; 20)$; approximation of the limiting mean number $\varphi(t)$ of customers in the system for $t \in[5,6]$.

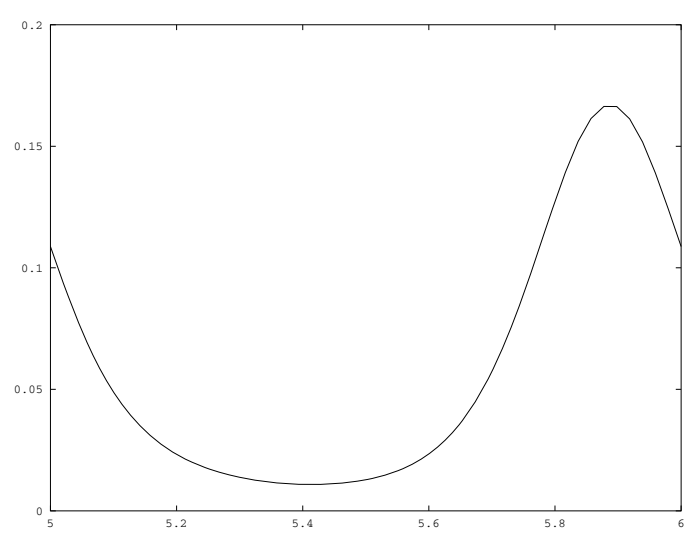

Fig. 9. Case (iii): the arrival intensity is $\lambda^{*}(t ; 20)$; approximation of the limiting probability $p_{0}(t)$ of the empty queue for $t \in[5,6]$.

of Zeifman et al. (2016b) and is related to optimization of "no-wait" probabilities.

\section{Acknowledgment}

The publication was financially supported by the Ministry of Education and Science of the Russian Federation (agreement no. 02.a03.21.0008) and by the Russian Foundation for Basic Research (projects no. 15-01-01698, 18-01-00309).

\section{References}

Almasi, B., Roszik, J. and Sztrik, J. (2005). Homogeneous finite-source retrial queues with server subject to breakdowns and repairs, Mathematical and Computer Modelling 42(5): 673-682.

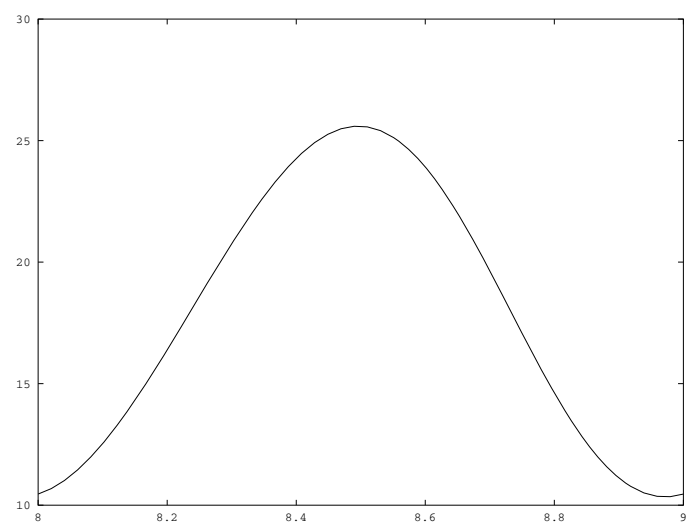

Fig. 10. Case (iii): the arrival intensity is $\lambda^{*}(t ; 50)$; approximation of the limiting mean number $\varphi(t)$ of customers in the system for $t \in[8,9]$.

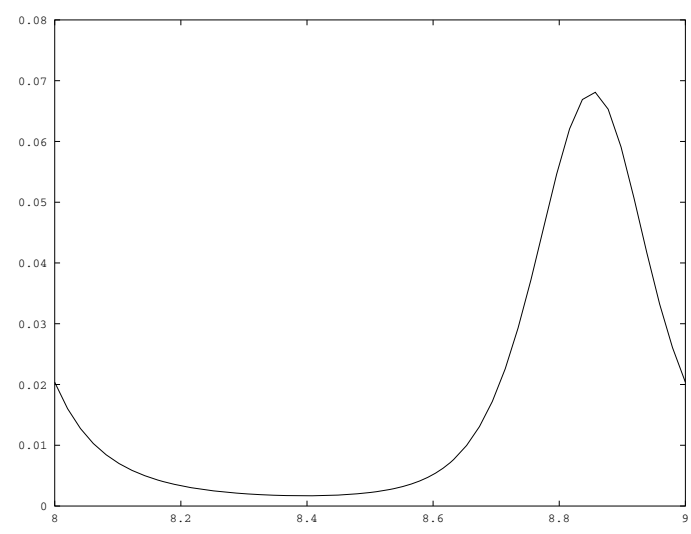

Fig. 11. Case (iii): the arrival intensity is $\lambda^{*}(t ; 50)$; approximation of the limiting probability $p_{0}(t)$ of the empty queue for $t \in[8,9]$.

Chen, A., Pollett, P., Li, J. and Zhang, H. (2010). Markovian bulk-arrival and bulk-service queues with state-dependent control, Queueing Systems 64(3): 267-304.

Daleckij, J. and Krein, M. (1974). Stability of Solutions of Differential Equations in Banach Space, American Mathematical Society, Providence, RI

Doorn, E.V., Zeifman, A. and Panfilova, T. (2010). Bounds and asymptotics for the rate of convergence of birth-death processes, Theory of Probability and Its Applications 54(1): 97-113.

Granovsky, B. and Zeifman, A. (2000). The $n$-limit of spectral gap of a class of birthdeath Markov chains, Applied Stochastic Models in Business and Industry 16(4): 235-248.

Granovsky, B. and Zeifman, A. (2004). Nonstationary queues: Estimation of the rate of convergence, Queueing Systems 46(3-4): 363-388.

Gudkova, I., Korotysheva, A., Zeifman, A., Shilova, G., Korolev, V., Shorgin, S. and Razumchik, R. (2016). Modeling and 


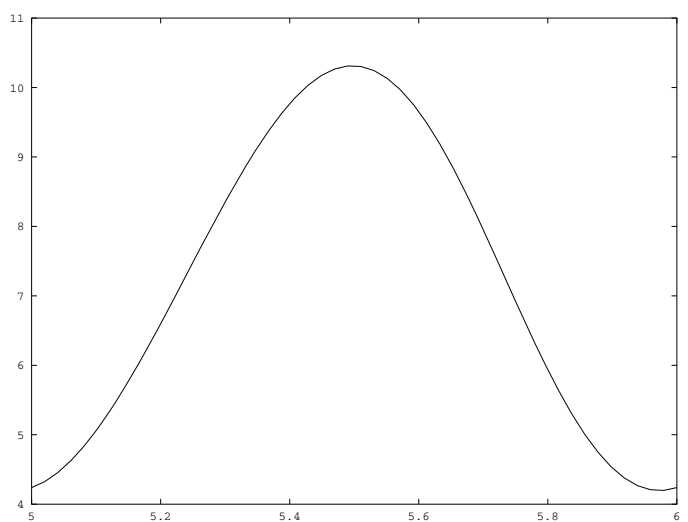

Fig. 12. Case (iv): the arrival intensity is $\lambda^{*}(t ; 20)$; approximation of the limiting mean number $\varphi(t)$ of customers in the system for $t \in[5,6]$.

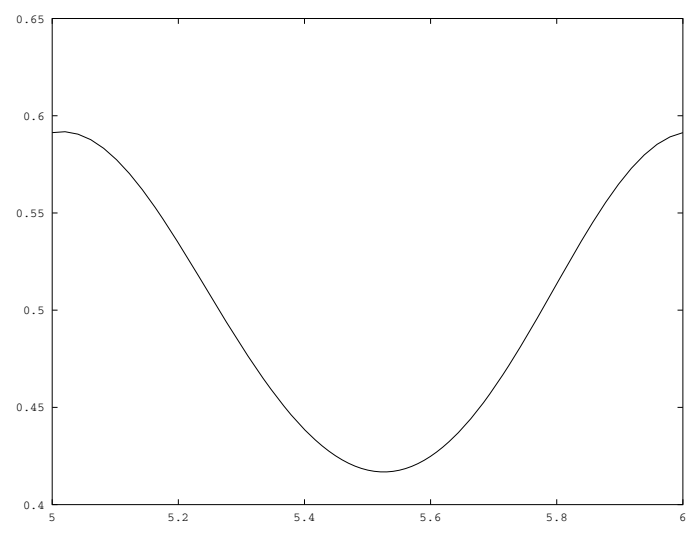

Fig. 13. Case (iv): the arrival intensity is $\lambda^{*}(t ; 20)$; approximation of the limiting probability $p_{0}(t)$ of the empty queue for $t \in[5,6]$.

analyzing licensed shared access operation for $5 \mathrm{~g}$ network as an inhomogeneous queue with catastrophes, 2016 8th International Congress on Ultra Modern Telecommunications and Control Systems and Workshops (ICUMT), Lisbon, Portugal, pp. 282-287.

Kamiński, M. (2015). Symbolic computing in probabilistic and stochastic analysis, International Journal of Applied Mathematics and Computer Science 25(4): 961-973, DOI: 10.1515/amcs-2015-0069.

Kartashov, N. (1985). Criteria for uniform ergodicity and strong stability of Markov chains with a common phase space, Theory of Probability and Mathematical Statistics 30(30): 71-89.

Kartashov, N. (1996). Strong Stable Markov Chains, VSP, Utrecht.

Kim, C., Dudin, A., Dudin, S. and Dudina, O. (2014). Analysis of an $M M A P / P H_{1}, P H_{2} / N / \infty$ queueing system operating in a random environment, International

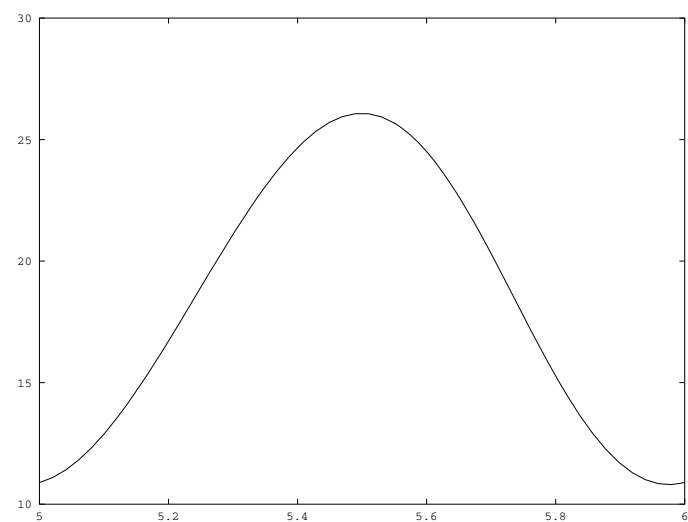

Fig. 14. Case (iv): the arrival intensity is $\lambda^{*}(t ; 50)$; approximation of the limiting mean number $\varphi(t)$ of customers in the system for $t \in[5,6]$.

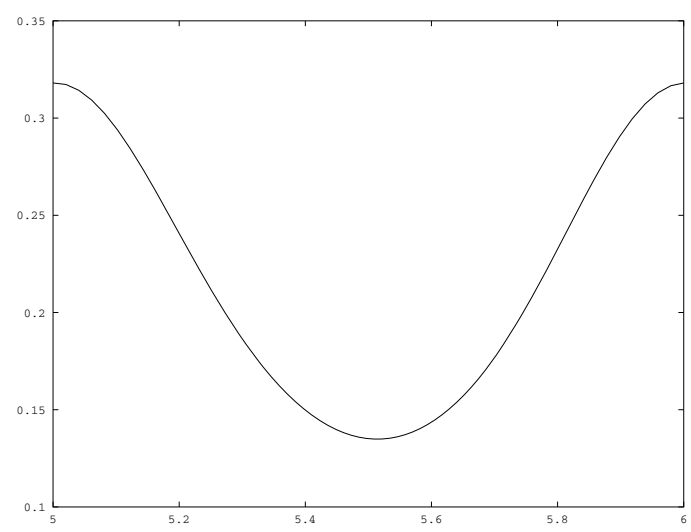

Fig. 15. Case (iv): the arrival intensity is $\lambda^{*}(t ; 50)$; approximation of the limiting probability $p_{0}(t)$ of the empty queue for $t \in[5,6]$.

Journal of Applied Mathematics and Computer Science 24(3): 485-501, DOI: 10.2478/amcs-2014-0036.

Li, J. and Zhang, L. (2016). Decay property of stopped Markovian bulk-arriving queues with c-servers, Stochastic Models 32(4): 674-686.

Mitrophanov, A. (2003). Stability and exponential convergence of continuous-time Markov chains, Journal of Applied Probability 40(4): 970-979.

Mitrophanov, A. (2004). The spectral GAP and perturbation bounds for reversible continuous-time Markov chains, Journal of Applied Probability 41(4): 1219-1222.

Mitrophanov, A. (2005a). Ergodicity coefficient and perturbation bounds for continuous-time Markov chains, Mathematical Inequalities \& Applications 8(1): 159-168.

Mitrophanov, A. (2005b). Sensitivity and convergence of uniformly ergodic Markov chains, Journal of Applied Probability 42(4): 1003-1014. 
Moiseev, A. and Nazarov, A. (2016a). Queueing network $M A P-(G I / \infty) K$ with high-rate arrivals, European Journal of Operational Research 254(1): 161-168.

Moiseev, A. and Nazarov, A. (2016b). Tandem of infinite-server queues with Markovian arrival process, Distributed Computer and Communication Networks: 18th International Conference, DCCN 2015, Moscow, Russia, pp. 323-333.

Nelson, R., Towsley, D. and Tantawi, A. (1987). Performance analysis of parallel processing systems, ACM SIGMETRICS Performance Evaluation Review 15(1): 93-94.

Satin, Y., Zeifman, A. and Korotysheva, A. (2013). On the rate of convergence and truncations for a class of Markovian queueing systems, Theory of Probability \& Its Applications 57(3): 529-539.

Schwarz, J., Selinka, G. and Stolletz, R. (2016). Performance analysis of time-dependent queueing systems: Survey and classification, Omega 63: 170-189.

Whitt, W. (1991). The pointwise stationary approximation for $M_{t} / M_{t} / s$ queues is asymptotically correct as the rates increase, Management Science 37(3): 307-314.

Whitt, W. (2015). Stabilizing performance in a single-server queue with time-varying arrival rate, Queueing Systems 81(4): 341-378.

Zeifman, A. (1995a). On the estimation of probabilities for birth and death processes, Journal of Applied Probability 32(3): 623-634.

Zeifman, A. (1995b). Upper and lower bounds on the rate of convergence for nonhomogeneous birth and death processes, Stochastic Processes and Their Applications 59(1): 157-173.

Zeifman, A. and Korolev, V. (2014). On perturbation bounds for continuous-time Markov chains, Statistics \& Probability Letters 88: 66-72.

Zeifman, A. and Korolev, V. (2015). Two-sided bounds on the rate of convergence for continuous-time finite inhomogeneous Markov chains, Statistics \& Probability Letters 103: 30-36.

Zeifman, A., Korolev, V., Satin, Y., Korotysheva, A. and Bening, V. (2014a). Perturbation bounds and truncations for a class of Markovian queues, Queueing Systems 76(2): 205-221.

Zeifman, A., Korotysheva, A., Korolev, V. and Satin, Y. (2016a). Truncation bounds for approximations of inhomogeneous continuous-time Markov chains, Probability Theory and Its Applications 61(3): 563-569, (in Russian).

Zeifman, A., Korotysheva, A., Satin, Y., Shilova, G., Razumchik, R., Korolev, V. and Shorgin, S. (2016b). Uniform in time bounds for "no-wait" probability in queues of $M_{t} / M_{t} / S$ type, Proceedings of the 30th European Conference on Modelling and Simulation, ECMS 2016, Regensburg, Germany, pp. 676-684.

Zeifman, A., Leorato, S., Orsingher, E., Satin, Y. and Shilova, G. (2006). Some universal limits for nonhomogeneous birth and death processes, Queueing Systems 52(2): 139-151.
Zeifman, A., Satin, Y., Korolev, V. and Shorgin, S. (2014b). On truncations for weakly ergodic inhomogeneous birth and death processes, International Journal of Applied Mathematics and Computer Science 24(3): 503-518, DOI: 10.2478/amcs-2014-0037.

Zeifman, A., Satin, Y. and Panfilova, T. (2013). Limiting characteristics for finite birthdeath-catastrophe processes, Mathematical Biosciences 245(1): 96-102.

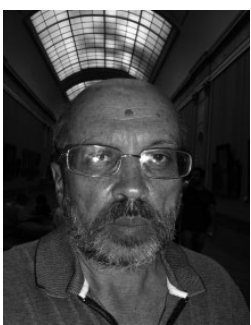

Alexander Zeifman is a Doctor of Science in physics and mathematics; a professor; the head of the Department of Applied Mathematics at Vologda State University; a senior scientist at the Institute of Informatics Problems, Federal Research Center on Computer Science and Control of the Russian Academy of Sciences; a principal scientist at the Institute of Socio-Economic Development of Territories of the Russian Academy of Sciences. His current research activities focus on inhomogeneous Markov chains and queueing theory.

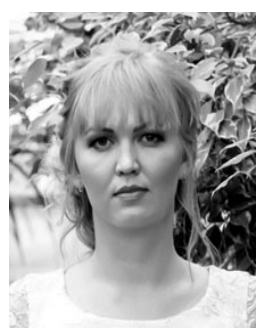

Anna Korotysheva is a Candidate of Science $(\mathrm{PhD})$ in physics and mathematics and a senior lecturer at Vologda State University. Her current research activities focus on queueing theory.

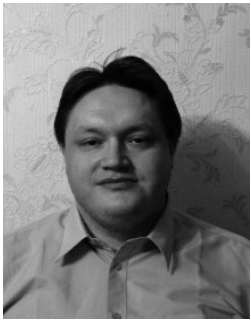

Yacov Satin is a Candidate of Science $(\mathrm{PhD})$ in physics and mathematics, and an associate professor at Vologda State University. His current research activities focus on inhomogeneous Markov chains and queueing theory.

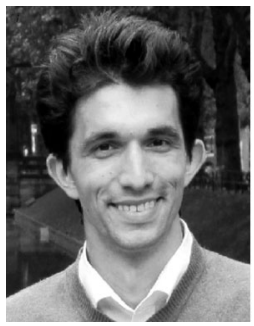

Rostislav V. Razumchik received his $\mathrm{PhD}$ degree in physics and mathematics in 2011. Since then, he has worked as a leading research fellow at the Institute of Informatics Problems of the Federal Research Center in Computer Science and Control of the Russian Academy of Sciences (FRC CSC RAS). Currently he also holds the associate professor position at Peoples' Friendship University of Russia (RUDN University). His present research activities are focused on queueing theory and its applications for performance evaluation of stochastic systems. 


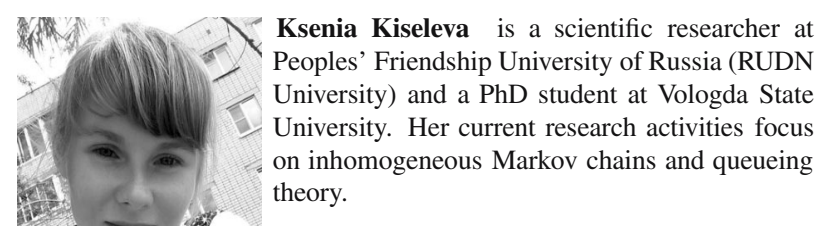

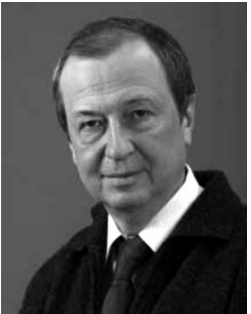

Victor Korolev is a Doctor of Science in physics and mathematics; a professor; the head of the Department of Mathematical Statistics, Faculty of Computational Mathematics and Cybernetics, M.V. Lomonosov Moscow State University; a leading scientist at the Institute of Informatics Problems of the Russian Academy of Sciences. His current research activities focus on stochastic models and queueing theory.

Received: 24 April 2017

Revised: 18 July 2017

Accepted: 9 August 2017 